NESMCQ18

Experimental investigations of local stochastic resistive switching in yttria stabilized zirconia film on a conductive substrate

To cite this article: D O Filatov et al J. Stat. Mech. (2020) 024005

View the article online for updates and enhancements.

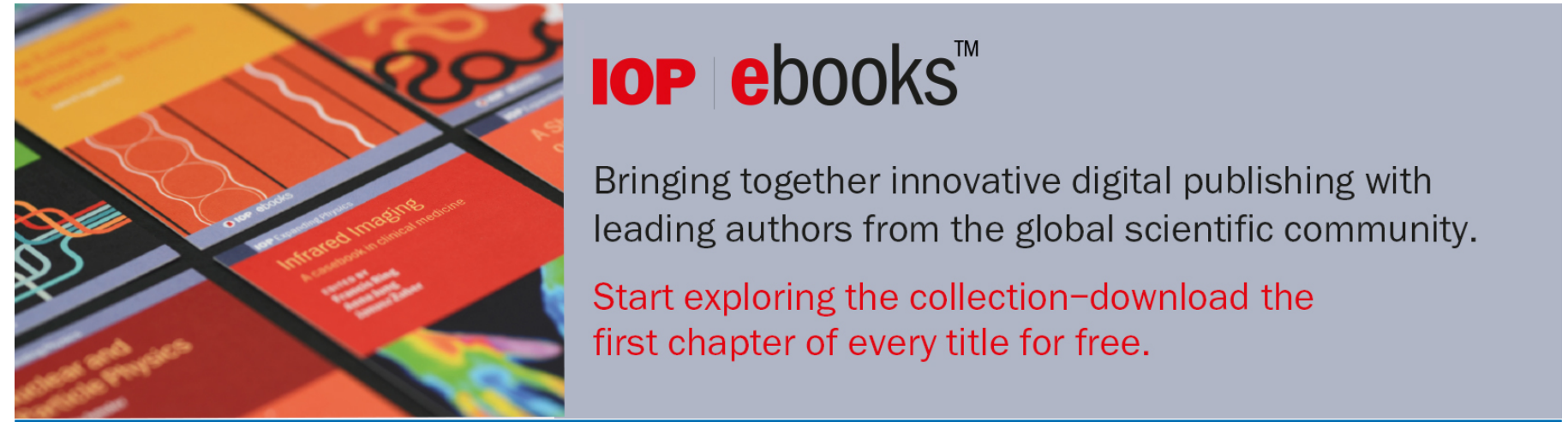

This content was downloaded from IP address 147.163 .1 .85 on $16 / 04 / 2020$ at $18: 00$ 


\title{
Experimental investigations of local stochastic resistive switching in yttria stabilized zirconia film on a conductive substrate
}

\author{
D O Filatov ${ }^{1}$, A S Novikov ${ }^{1}$, V N Baranova ${ }^{1}$, \\ D A Antonov ${ }^{1}$, A V Kruglov ${ }^{1}$, I N Antonov ${ }^{1}$, \\ A V Zdoroveyshchev ${ }^{1}, M$ N Koryazhkina ${ }^{1,2}$, \\ O N Gorshkov ${ }^{1}$, A A Dubkov ${ }^{1}$, A Carollo ${ }^{1,2}$ \\ and B Spagnolo ${ }^{1,2,3}$ \\ ${ }^{1}$ Lobachevskii State University of Nizhnii Novgorod, Gagarin Ave. 23, 603950 \\ Nizhnii Novgorod, Russia \\ 2 Dipartimento di Fisica e Chimica-Emilio Segrè, Group of Interdisciplinary \\ Theoretical Physics, Università di Palermo and CNISM, Unità di Palermo, \\ Viale delle Scienze, Edificio 18, I-90128 Palermo, Italy \\ ${ }^{3}$ Istituto Nazionale di Fisica Nucleare, Sezione di Catania, Via S. Sofia 64, \\ 95123 Catania, Italy \\ E-mail: dmitry_filatov@inbox.ru,nsv3333@yandex.ru,vera.baranova00@mail. \\ ru, antonov@phys.unn.ru,krualex@yandex.ru, ivant@nifti.unn.ru,zdorovei@ \\ gmail.com,mahavenok@mail.ru,gorshkov@nifti.unn.ru, dubkov@rf.unn.ru, \\ angelo.carollo@unipa.it and bernardo.spagnolo@unipa.it
}

Received 12 October 2019

Accepted for publication 9 December 2019

Published 24 February 2020

Online at stacks.iop.org/JSTAT/2020/024005

https://doi.org/10.1088/1742-5468/ab69ff

\begin{abstract}
We report on the results of the experimental investigations of the local resistive switching (RS) in the contact of a conductive atomic force microscope (CAFM) probe to a nanometer-thick yttria stabilized zirconia (YSZ) film on a conductive substrate under a Gaussian noise voltage applied between the probe and the substrate. The virtual memristor was found to switch randomly between the low resistance state and the high resistance state as a random telegraph signal (RTS). The potential profile of the virtual memristor calculated from its response to the Gaussian white noise shows two local minima, which is peculiar of a bistable nonlinear system.
\end{abstract}


Keywords: diffusion, stochastic particle dynamics, fluctuation phenomena, brownian motion

\section{Contents}

1. Introduction

3. Results and discussion

4. Conclusions
Acknowledgments $\ldots \ldots \ldots \ldots \ldots \ldots \ldots \ldots \ldots \ldots \ldots \ldots \ldots \ldots \ldots \ldots \ldots \ldots \ldots \ldots \ldots \ldots \ldots \ldots \ldots \ldots \ldots \ldots \ldots \ldots$

$\begin{array}{ll}\text { References } & 9\end{array}$

\section{Introduction}

In recent years, the investigations of resistive switching (RS) have attracted much attention [1]. The effect of RS consists in the bistable (or multistable) switching of the resistance of thin nanometric dielectric films sandwiched between two conductive electrodes under the external voltage [2]. In the context of nanotechnology, the production of nanostructures by diffusion is a widely studied topic [3-5]. The electronic devices, the functioning of which is based on the RS are called memristors [6]. The memristors are considered to be promising for application in the next generation non-volatile computer memory (resistive random access memory, ReRAM) [7], in the neuromorphic computer systems [8], etc.

Today's understanding of the RS mechanism in the metal oxides is based on the concept of the formation of the conductive filaments consisting of the oxygen vacancies between the conductive electrodes in the electric field between the electrodes (shortcutting these ones) in so-called forming process [9]. The switching of a memristor from the low resistance state (LRS) to the high resistance state (HRS) is achieved by the rapture of the filament by a voltage pulse (so-called RESET process). The filament can be restored by a voltage pulse of the opposite polarity that results in the switching from the HRS back to LRS (so-called SET process).

It should be emphasized here that the RS is a stochastic process. In fact, the intrinsic fluctuations in structure, chemistry, physical values and switching times, which can occur on multiple lengths and time scales during switching events, are of a stochastic nature and this causes the stochastic of resistive switching [10-14]. Moreover, this is also related to typical small size of the filament cross-section, which falls into the range from 1 to $10 \mathrm{~nm}$ [15]. Usually, the rapture and restoring of the filaments in oxides takes place randomly at its interface with one of the electrodes through the jumps of the $\mathrm{O}^{2-}$ ions via the oxygen vacancies in a small volume (several cubic nanometers) near the filament tip. A very limited (countable) number of the $\mathrm{O}^{2-}$ ions (or the oxygen vacancies) are involved into the RS [16]. The aforementioned factors, from one switching cycle to another (so-called cycle-to-cycle or C2C scatter), lead to a wide dispersion of 
Experimental investigations of local stochastic resistive switching in yttria stabilized zirconia film

the memristor parameter values, such as the switching voltage from HRS to LRS $V_{\mathrm{SET}}$ and back to $V_{\text {RESET }}$, etc, which can reach one hundred percent [17]. The natural dispersion of the memristor parameters, which is a peculiar property of the RS, currently limits the application of the memristors [18]. For example, the C2C scatter of $V_{\text {SET }}$ and $V_{\text {RESET }}$ values makes it difficult to correctly select the logical levels in the design of strongly ReRAM devices [19].

The memristor can be treated as a bistable (in the simplest case) or multistable nonlinear system. It should be noted that the coexistence of several metastable states for a given set of parameters has been observed in many natural and experimental systems in such different areas as laser and semiconductor physics, chemistry, ecology, neuroscience, climate dynamics (see the review [20] and references therein). Multistable systems are characterized by a high degree of complexity in their behavior due to the 'interaction' among the attractors and can be describe by the methods of statistical physics. Specifically, in such systems, the well-known stochastic resonance phenomenon [21-24] can occur, where noise plays a constructive role [25-30]. In fact, there are many known examples of classical and quantum physics, in which the synergistic cooperation between the non-linearity of the system and the environmental noise gives rise to counterintuitive dynamical behaviors [31-48].

Within the framework of the above-mentioned approach, from a theoretical point of view a memristor can be described by a two-well (or more complex) potential profile. Several authors attempted to apply this approach. Moreover, some phenomena inherent to the stochastic multistable systems have been observed experimentally in memristors. In particular, the beneficiary role of adding the white noise to the switching pulses on the stability of the RS parameters have been observed in [41]. However, it is still unclear whether this approach is applicable for the description of memristors.

In the present study, the response of the contact between a conductive atomic force microscope (CAFM) probe and the yttria stabilized zirconia (YSZ) film on a conductive substrate to an external digitally synthesized noise signal is investigated. $\mathrm{YSZ}_{\mathrm{ZrO}}(\mathrm{Y})$ is considered to be a promising functional material for the memristive applications due to a high anion mobility [49]. CAFM has been proven to be a powerful tool for studying the local RS at the nanometer scale $[50,51]$. In such experiments, the CAFM probe contact to a dielectric film on a conductive substrate can be treated as a nanometer-sized virtual memristor. Typical size of the contact area between the metal-coated CAFM probe tip and YSZ films $D_{p}$ is $<10 \mathrm{~nm}$ at reasonable loading forces $F_{n} \sim 1 \mathrm{nN}$ [52] that matches the expected sizes of memristors in future ReRAM devices [53]. So far, CAFM probe contact to the dielectric film surface on a conductive substrate appears to be a good model system for studying the local RS at the nanometer scale [54]. Particularly, the electrical properties of individual filaments can be studied using CAFM.

The goal of the present work is the search for the fundamental phenomena featuring the memristor as a multistable nonlinear system. In the presence of a Gaussian white noise signal, we have observed the randomly switching of the virtual memristor between the HRS and LRS as a random telegraph signal (RTS). The potential profile extracted from the CAFM probe current waveforms shows two local minima corresponding to HRS and LRS states of the virtual memristor. These observations manifest the fundamental intrinsic properties of the memristor as a bistable nonlinear stochastic system. 
The paper is organized as follows. In the next section, we describe the details of the sample preparation as well as of the experimental procedure on studying the response of the CAFM probe-to-sample contact to an external digitally synthesized Gaussian white noise signal. The results of experiments are presented and analyzed in section 3 . Section 4 is devoted to concluding remarks.

\section{Experimental}

The YSZ films of $\approx 4 \mathrm{~nm}$ in thickness are deposited by radio-frequency magnetron sputtering using Torr International ${ }^{\circledR} 2$ G1-1G2-EB4-TH1 vacuum setup for thin film deposition. The sample cross-section is shown schematically in figure 1.

Standard factory-made $\mathrm{Si}(001)$ substrates with pre-deposited $\mathrm{SiO}_{2}(500 \mathrm{~nm}), \mathrm{Ti}$ $(25 \mathrm{~nm})$ and $\operatorname{TiN}(25 \mathrm{~nm})$ layers are used. The substrate temperature when depositing YSZ is $300{ }^{\circ} \mathrm{C}$. The pressure of $\mathrm{Ar}-\mathrm{O}_{2}\left(50: 50 \%\right.$ mol.) gas is $\sim 10^{-2}$ Torr. The molar fraction of the stabilizing oxide $\mathrm{Y}_{2} \mathrm{O}_{3}$ in the pressed powder target material is $\approx 0.12$. The RS is investigated in ultra high vacuum (UHV) environment (the base residual gas pressure $\sim 10^{-10}$ Torr) using Omicron ${ }^{\circledR}$ UHV AFM/STM LF1 installed into Omicron ${ }^{\circledR}$ MultiProbe ${ }^{\mathrm{TM}}$ RM UHV setup. NT-MD ${ }^{\circledR}$ NSG-11 DCP ${ }^{\mathrm{TM}}$ probes covered by diamondlike coating are used. The schematic representation of the experimental setup is shown in figure 1 . The bias voltage $V_{\mathrm{g}}$ applied between the CAFM probe and the TiN conductive layer is supplied by the digital-to-analog converter (DAC) of Lcard $^{\circledR}$ E502 PC-controlled ADC/DAC unit employed as an external programmable voltage source. First, the AFM probe is brought into contact with the YSZ film surface, and the adaptive forming is performed by supplying the forming voltage $V_{\text {form }}=5-6 \mathrm{~V}$ between the AFM probe and the sample until the probe current $I_{\mathrm{t}}$ reaches the preset threshold value 10-15 nA. Afterwards, the cyclic current-voltage ( $I-V)$ curves of the CAFM probe-tosample contact (virtual memristor) $I_{\mathrm{t}}\left(V_{\mathrm{g}}\right)$ are measured by sweeping the ramp voltage $V_{\mathrm{g}}$ from $V_{\min }<V_{\mathrm{RESET}} \approx-6 \mathrm{~V}$ up to $V_{\max }>V_{\mathrm{SET}} \approx+5 \mathrm{~V}$ and back down to $V_{\min }$ and so forth in order to ensure the proper RS. The ramp voltage sweep rate is 1 to $5 \mathrm{~V} \mathrm{~s}^{-1}$.

In order to investigate the impact of noise on the virtual memristor response, a digitally synthesized external noise signal with predefined magnitude is applied between the CAFM probe and the substrate using Lcard ${ }^{\circledR}$ ADC/DAC unit control software. The digitally synthesized Gaussian white noise signal is generated using a random number generator of ADSViewer-2 (v.015) software module [55] and is saved in a control PC. ADSViewer-2 software system is running under LabVIEW ${ }^{\circledR} 6.1$ software system and allows generating the series of random numbers with Gaussian distribution utilizing either the summation of several series of random numbers with uniform distribution or a nonlinear transformation of a series of random numbers with uniform distribution. The noise data array contains $2^{16}$ random numbers. Before measurements, the noise data array is read into the random access memory (RAM) of the control PC. The magnitude of the noise signal is defined by scaling the original noise data and characterized by the output voltage standard deviation $\sigma_{\mathrm{V}}$. Finally, a constant offset voltage $V_{\text {offset }}$ is added to the noise data. The resulting signal data array is loaded into the RAM buffer of Lcard $^{\circledR}$ E502 ADC/DAC unit via the USB interface. In the experiment, the noise 
Experimental investigations of local stochastic resistive switching in yttria stabilized zirconia film

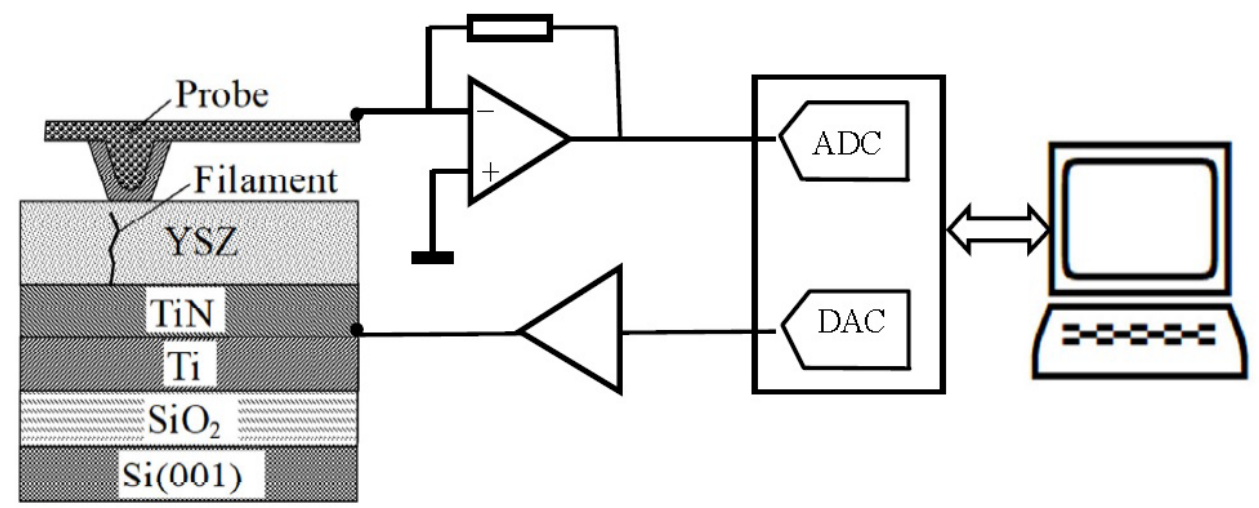

Figure 1. Schematic representation of the experimental setup.

signal is played back, sent to the DAC of Lcard ${ }^{\circledR}$ E502 ADC/DAC unit, in an endless cycle with predefined bit rate. The playback is performed by the digital signal processor (DSP) of Lcard ${ }^{\circledR}$ E502 ADC/DAC unit in the remote mode. The maximum bit rate is $10^{6}$ numbers per second, which corresponds to the white noise spectrum bandwidth from 0 to $1 \mathrm{MHz}$. The signal from the DAC output of Lcard ${ }^{\circledR}$ E502 ADC/DAC unit is amplified up to the maximum magnitude of $10 \mathrm{~V}$ by a wide bandpass dc operation voltage amplifier and supplied to the external bias voltage input of Omicron ${ }^{\circledR}$ UHV AFM/ STM LF1. In order to obtain a wide-band external white noise signal between the CAFM probe and the sample, the original in-vacuum operation amplifier in the STM preamplifier (current-voltage converter) of Omicron ${ }^{\circledR}$ UHV AFM/STM LF1 is replaced by an external (ex-vacuum) preamplifier with the bandpass of 0-7 MHz. The probe current $I_{\mathrm{t}}$ signal is taken from the 'Tip current' output of Omicron ${ }^{\circledR} \mathrm{SCALA}^{\mathrm{TM}} \mathrm{AFM} /$ STM electronic control unit and supplied to the analog-to-digital converter (ADC) of Lcard $^{\circledR}$ E502 ADC/DAC unit operated in the buffering mode. The maximum sampling rate of the $\mathrm{ADC}$ is $2 \mathrm{MHz}$.

In the experiment, the waveforms $I_{\mathrm{t}}(t)$ are recorded subject to the parameters of the input noise signal $\sigma_{\mathrm{V}}$ and $V_{\text {offset }}$.

\section{Results and discussion}

Figure 2 shows the typical cyclic $I-V$ curve of the CAFM probe contact to the YSZ film surface. A pronounced hysteresis typical for the bipolar RS has been observed. One can note different values of $V_{\mathrm{SET}} \approx 5 \mathrm{~V}$ and $V_{\text {RESET }} \approx-6 \mathrm{~V}$ owing to the asymmetry of the virtual memristor structure: the functional YSZ layer contacted with chemically active TiN conductive layer from the bottom side and to the chemically inert DCP coating of the CAFM probe at the surface.

In figure 3 is shown the probe current waveforms $I_{\mathrm{t}}(t)$ recorded at $V_{\text {offset }}=-1 \mathrm{~V}$ and different values of $\sigma_{\mathrm{V}}$, corresponding to different noise magnitude, and the respective probability density functions (PDFs) of the probe current distributions.

At $\sigma_{\mathrm{V}}=0.3 \mathrm{~V}$, for values of $V_{\mathrm{g}}$ within the linear region in the $I-V$ curve of the virtual memristor, the distribution of $I_{\mathrm{t}}$ has an almost Gaussian form as that of the input 
Experimental investigations of local stochastic resistive switching in yttria stabilized zirconia film

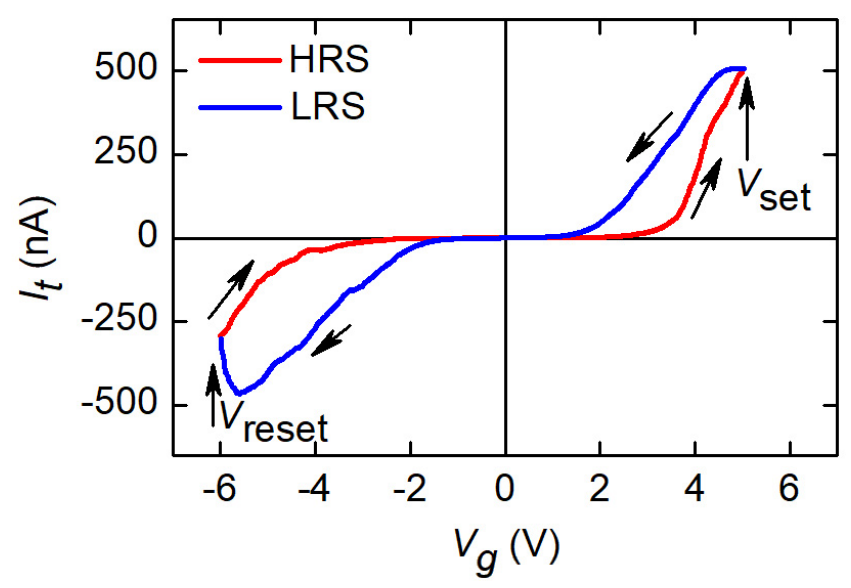

Figure 2. Typical $I-V$ curve of the CAFM probe-to-sample contact.
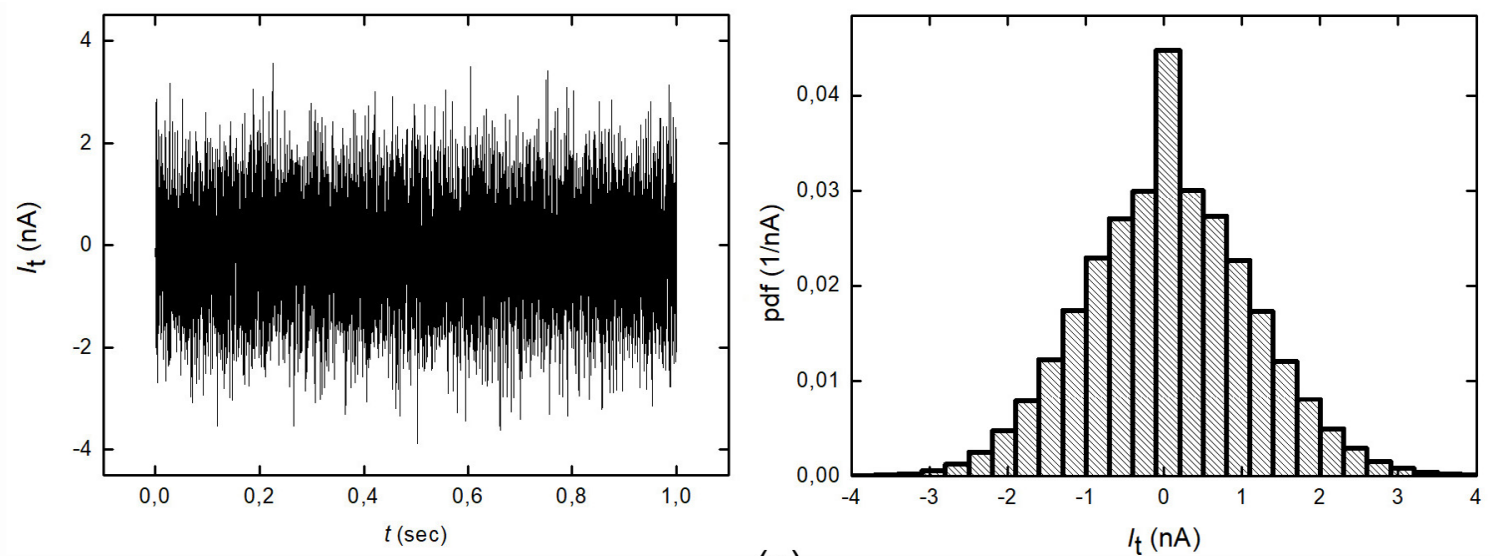

(a)
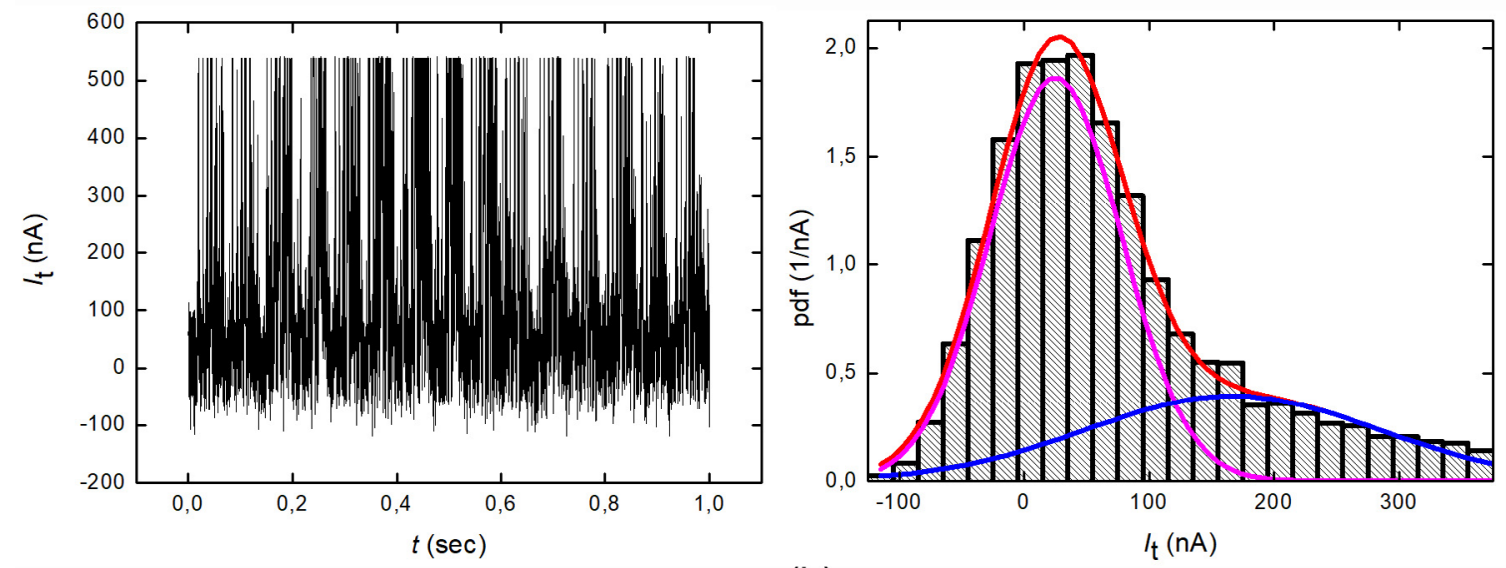

(b)

Figure 3. The waveforms (left) and PDFs (right) of the CAFM probe current $I_{\mathrm{t}}$. $V_{\text {offset }}=-1 \mathrm{~V} \cdot \sigma_{\mathrm{V}}, \mathrm{V}: 0.3(\mathrm{a}) ; 0.9(\mathrm{~b})$.

voltage $V_{\mathrm{g}}$ (figure $3(\mathrm{a})$ ). In this case, the virtual memristor acts as a linear resistor (in the HRS) and the distribution of $I_{\mathrm{t}}$ simply reflects that of $V_{\mathrm{g}}$. Slight deviation of $I_{\mathrm{t}}$ from the Gaussian shape can be attributed to the nonlinearity of the $I-V$ curve of the virtual memristor. In the presence of the digitally synthesized external noise signal, 


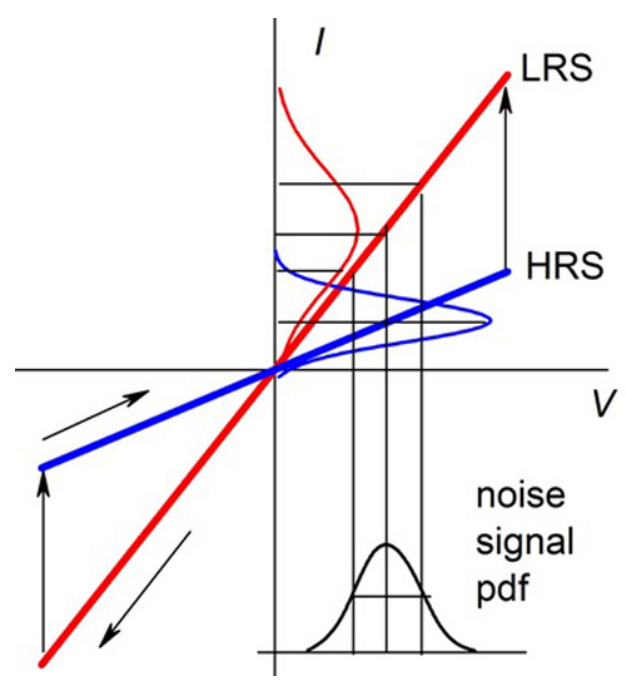

Figure 4. Analysis of the current response of the memristor to a Gaussian white noise voltage.
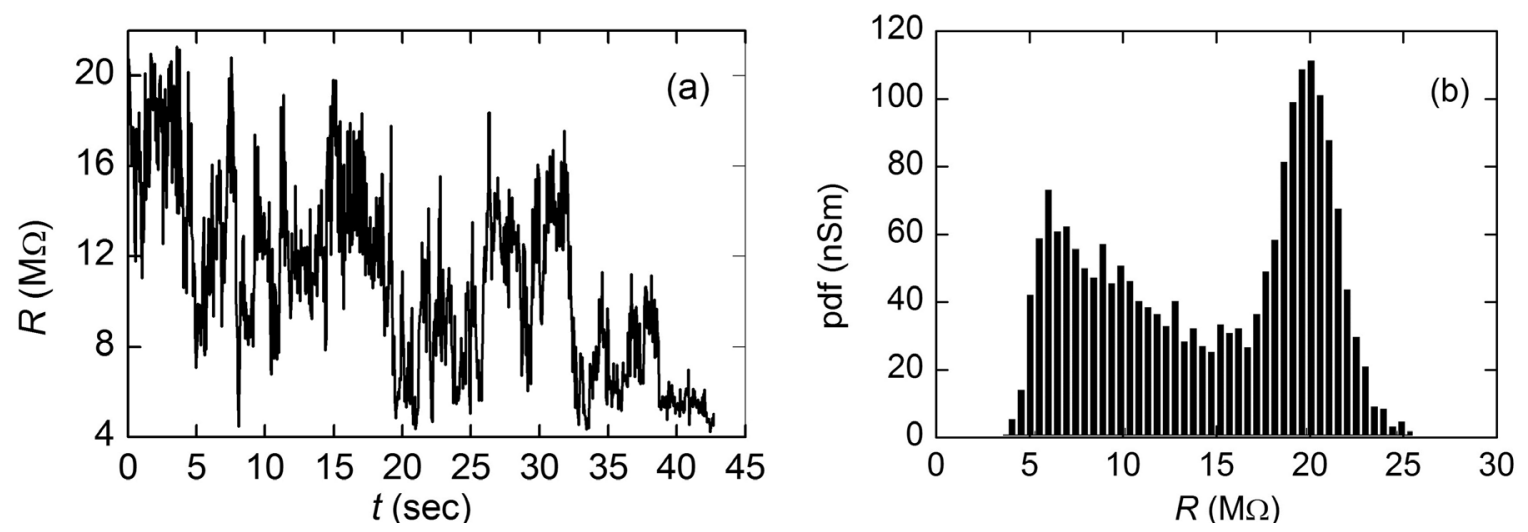

Figure 5. Waveform (a) and PDF (b) of the virtual memristor resistance $R$ calculated according to equation (1) in the noise-induced RS mode.

when $\sigma_{\mathrm{V}}=0.9 \mathrm{~V}$, the virtual memristor switches between the HRS and LRS randomly as a RTS (see figure 3(b)). Such a behavior is typical for bistable systems. So far, the experimental results obtained in the present study point to the applicability of the formalism used in the statistical physics to describe the role of the noise on the nonlinear multistable systems for understanding the stochastic resistive switching of memristors. The noise-induced RS was observed in the range of $V_{\text {offset }}$ from $-1 \mathrm{~V}$ to $-0.5 \mathrm{~V}$. Note that this range of $V_{\text {offset }}$ corresponds to the mean value of $V_{\text {SET }}$ and $V_{\text {RESET }}$ in the cyclic $I-V$ curve of the CAFM probe-to-sample contact (see figure 2).

In order to analyze the distribution of $I_{\mathrm{t}}$, let us consider a cyclic $I-V$ curve of an ideal memristor presented in figure 4. Here the branches corresponding to the HRS and LRS are represented by linear functions, that is the memristor is represented as a linear switching device. Let us consider a random voltage $V$ with Gaussian distribution applied to the memristor. In the HRS, the distribution of the current through the memristor $I$ will also be Gaussian. In the LRS, the distribution of $I$ will be Gaussian too, but its width will be greater than in the HRS. If the memristor switches between HRS and LRS many times within an observation period of time, the resulting distribution of 
Experimental investigations of local stochastic resistive switching in yttria stabilized zirconia film

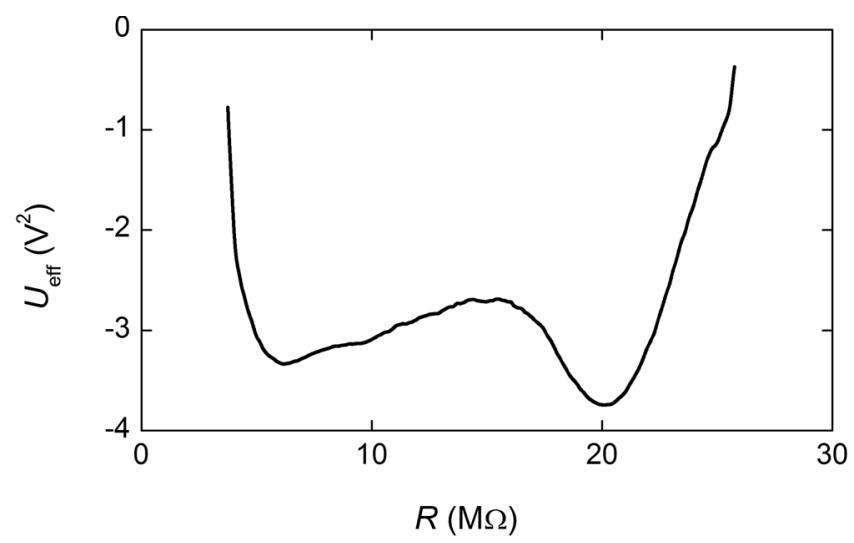

Figure 6. Potential profile of the virtual memristor reconstructed from the virtual memristor resistance waveform $R(t)$ shown in figure $5(\mathrm{a})$.

$I$ would be a superposition of two Gaussian functions with respective widths. Indeed, the experimental PDF of $I_{\mathrm{t}}$ can be approximated by a superposition of two Gaussian functions as shown in figure 3(b), where the PDFs for LRS (purple curve), HRS (blue curve) and superposition of the previous curves (red curve) are shown. In the case when a non-zero bias voltage $V_{\text {offset }}$ is added to the Gaussian noise signal, the centers of the corresponding Gaussian distributions of $I$ in the HRS and in the LRS would be shifted with respect to each other as shown in figure 4 . This effect explains the asymmetry of the PDF of $I_{\mathrm{t}}$ shown in figure 3(b). If $V_{\text {offset }}=0$, the centers of both Gaussian distributions will take place at $I=0$, and the resulting distribution of $I$ will be symmetric.

Taking into account all the above, in the present study the state of the virtual memristor, at given time $t$, is characterized by the mean resistance $R(t)=\sigma_{\mathrm{V}} / \sigma_{\mathrm{I}}(t)$ where

$$
\sigma_{\mathrm{I}}(t)=\sqrt{\frac{\sum_{i=1}^{M}\left(I_{i}-\overline{I_{\mathrm{t}}}\right)^{2}}{M(M-1)}}
$$

is the standard deviation of $I$ calculated for a short time period $T_{a} \ll \tau, \tau$ being the minimum lifetime of the memristor in LRS or in HRS, $\overline{I_{\mathrm{t}}}$ is the averaged current over the time period $T_{a}$, and $M=100$ is the number of points in the time series of $I_{\mathrm{t}}$ measured within the period $T_{a}$. As one can see in figure $5(\mathrm{a}), R(t)$ changes in time as a RTS: the virtual memristor switches between two metastable states, which is reflected in the PDF of $R$ plotted in figure 5(b) where two peaks corresponding to HRS and LRS are observed.

In figure 6 , a potential profile of the virtual memristor, calculated for the waveform $R(t)$ shown in figure 5 (a) by standard procedure $[56,57]$, is shown. The potential profile manifest two minima corresponding to the numbers of switching levels observed in the $R(t)$ waveform and the number of peaks in the PDF of $R$ in figures 5(a) and (b), respectively.

\section{Conclusions}

In the present study, we have studied experimentally the response of a CAFM probe contact to an YSZ film on a conductive substrate, a virtual memristor, to an external 
Experimental investigations of local stochastic resistive switching in yttria stabilized zirconia film

Gaussian white noise signal. A stochastic switching of the virtual memristor between two metastable resistance states in the RTS mode under the noise signal and constant offset has been observed. The potential profile extracted from the waveform of the resistance of the virtual memristor manifests two local minima corresponding to the virtual switching levels of the memristor resistance observed in the experiment. The experimental results obtained highlight the applicability of the physical statistical formalism usually applied to the description of the behavior of nonlinear multistable systems to the description of the memristors.

\section{Acknowledgments}

This work was supported by the Government of the Russian Federation, Agreement No. 074-02-2018-330 (2).

\section{References}

[1] Ielmini D and Waser R 2016 Resistive Switching: from Fundamentals of Nanoionic Redox Processes to Memristive Device Applications (Weinheim: Wiley) p 784

[2] Waser R and Aono M 2007 Nanoionics-based resistive switching memories Nat. Mater. 6833

[3] Pankratov E L and Spagnolo B 2005 Optimization of impurity profile for p-n-junction in heterostructures Eur. Phys. J. B 4615

[4] Cheng C, Li W, Wong T-L, Ho M K, Fung K K and Wang N $2011 \mathrm{Zn}_{2} \mathrm{TiO}_{4}-\mathrm{ZnO}$ nanowire axial heterostructures formed by unilateral diffusion J. Phys. Chem. C 11578

[5] Strukov D B, Alibart F and Williams R S 2012 Thermophoresis/diffusion as a plausible mechanism for unipolar resistive switching in metal-oxide-metal memristors Appl. Phys. A 107509

[6] Strukov D B, Snider G S, Stewart D R and Williams R S 2008 The missing memristor found Nature 80453

[7] Ouyang J 2016 Emerging Resistive Switching Memories (Berlin: Springer) p 93

[8] James A 2018 Memristor and Memristive Neural Networks (London: IntechOpen) p 314

[9] Riess I 2017 Review of mechanisms proposed for redox based resistive switching structures J. Electroceram 3961

[10] Yu S, Guan X and Wong H-S P 2011 On the stochastic nature of resistive switching in metal oxide RRAM: physical modeling, Monte Carlo simulation, and experimental characterization Proc. IEEE Int. Electron Devices Meeting pp 17.3.1-4

[11] Li Q, Khiat A, Salaoru I, Xu H and Prodromakis T 2014 Stochastic switching of $\mathrm{TiO}_{2}$-based memristive devices with identical initial memory states Nanoscale Res. Lett. 91

[12] Hamilton T J, Afshar S, van Schaik A and Tapson J 2014 Stochastic electronics: a neuro-inspired design paradigm for integrated circuits Proc. IEEE 102 843-59

[13] Naous R, Al-Shedivat M and Salama K N 2016 Stochasticity modeling in memristors IEEE Trans. Nanotechnol. 1515

[14] Hull R et al 2018 Stochasticity in materials structure, properties and processing-a review Appl. Phys. Rev. 5011302

[15] Park G S et al 2013 In situ observation of filamentary conducting channels in an asymmetric $\mathrm{Ta}_{2} \mathrm{O}_{5-x} /$ $\mathrm{TaO}_{2-x}$ bilayer structure Nat. Commun. 42382

[16] Parreira P, Paterson G W, McVitie S and MacLaren D A 2016 Stability, bistability and instability of amorphous $\mathrm{ZrO}_{2}$ resistive memory devices J. Phys. D: Appl. Phys. 49095111

[17] Kim W, Menzel S, Wouters D J, Guo Y, Robertson J, Roesgen B, Waser R and Rana V 2016 Impact of oxygen exchange reaction at the ohmic interface in $\mathrm{Ta}_{2} \mathrm{O}_{5}$-based ReRAM devices Nanoscale 817774

[18] Yi W, Savel'ev S E, Medeiros-Ribeiro G, Miao F, Zhang M-X, Yang J J, Bratkovsky A M and Williams R S 2016 Quantized conductance coincides with state instability and excess noise in tantalum oxide memristors Nat. Commun. 711142

[19] Ielmini D 2016 Resistive switching memories based on metal oxides: mechanisms, reliability and scaling Semicond. Sci. Technol. 31063002

[20] Feudel U 2008 Complex dynamics in multistable systems Int. J. Bifurcation Chaos 181607 
Experimental investigations of local stochastic resistive switching in yttria stabilized zirconia film

[21] Mantegna R N and Spagnolo B 1995 Stochastic resonance in a tunnel diode in the presence of white or coloured noise Nuovo Cimento D 17873

[22] Lanzara E, Mantegna R N, Spagnolo B and Zangara R 1997 Experimental study of a nonlinear system in the presence of noise: the stochastic resonance Am. J. Phys. 65341

[23] Mantegna R N, Spagnolo B and Trapanese M 2001 Linear and nonlinear experimental regimes of stochastic resonance Phys. Rev. E 63011101

[24] Valenti D, Schimansky-Geier L, Sailer X and Spagnolo B 2006 Moment equations for a spatially extended system of two competing species Eur. Phys. J. B 50199

[25] Nicolis C 2010 Stochastic resonance in multistable systems: the role of intermediate states Phys. Rev. E 82011139

[26] La Barbera A and Spagnolo B 2002 Spatio-temporal patterns in population dynamics Physica A 314120

[27] Valenti D, Fiasconaro A and Spagnolo B 2004 Pattern formation and spatial correlation induced by the noise in two competing species Acta Phys. Pol. B 351481

[28] Agudov N V, Krichigin A V, Valenti D and Spagnolo B 2010 Stochastic resonance in a trapping overdamped monostable system Phys. Rev. E 81051123

[29] Nicolis C 2012 Stochastic resonance in multistable systems: the role of dimensionality Phys. Rev. E 86011133

[30] Han D, Su X and Shi P 2018 Stochastic resonance in multi-stable system driven by Lévy noise Chin. J. Phys. 561559

[31] Fiasconaro A, Valenti D and Spagnolo B 2004 Nonmonotonic behavior of spatiotemporal pattern formation in a noisy Lotka-Volterra system Acta Phys. Pol. B 351491

[32] Valenti D, Tranchina L, Brai M, Caruso A, Cosentino C and Spagnolo B 2008 Environmental metal pollution considered as noise: effects on the spatial distribution of benthic foraminifera in two coastal marine areas of Sicily (Southern Italy) Ecol. Mod. 213449

[33] Chichigina O, Valenti D and Spagnolo B 2005 A simple noise model with memory for biological systems Fluctuation Noise Lett. 5 L243

[34] Chichigina O A, Dubkov A A, Valenti D and Spagnolo B 2011 Stability in a system subject to noise with regulated periodicity Phys. Rev. E 84021134

[35] Consiglio A, Carollo A and Zenios S A 2016 A parsimonious model for generating arbitrage-free scenario trees Quantum Financ. 16201

[36] Spagnolo B, Carollo A and Valenti D 2018 Enhancing metastability by dissipation and driving in an asymmetric bistable quantum system Entropy 20226

[37] Falci G, La Cognata A, Berritta M, D’Arrigo A, Paladino E and Spagnolo B 2013 Design of a Lambda system for population transfer in superconducting nanocircuits Phys. Rev. B 87214515

[38] Spagnolo B, Carollo A and Valenti D 2018 Stabilization by dissipation and stochastic resonant activation in quantum metastable systems Eur. Phys. J.: Spec. Top. 227379

[39] Valenti D, Magazzù L, Caldara P and Spagnolo B 2015 Stabilization of quantum metastable states by dissipation Phys. Rev. B 91235412

[40] Stotland A and Di Ventra M 2012 Stochastic memory: memory enhancement due to noise Phys. Rev. E 85011116

[41] Patterson G A, Fierens P I and Grosz D F 2013 On the beneficial role of noise in resistive switching Appl. Phys. Lett. 103074102

[42] Valenti D, Carollo A and Spagnolo B 2018 Stabilizing effect of driving and dissipation on quantum metastable states Phys. Rev. A $\mathbf{9 7} 042109$

[43] Carollo A, Spagnolo B and Valenti D 2018 Uhlmann curvature in dissipative phase transitions Sci. Rep. 89852

[44] Carollo A, Spagnolo B and Valenti D 2018 Symmetric logarithmic derivative of fermionic gaussian states Entropy 20485

[45] Dubkov A A and Spagnolo B 2008 Verhulst model with Lévy white noise excitation Eur. Phys. J. B 65361

[46] Spagnolo B and Valenti D 2008 Volatility effects on the escape time in financial market models Int. J. Bifurcation Chaos 182775

[47] Spagnolo B, Guarcello C, Magazzù L, Carollo A, Persano Adorno D and Valenti D 2017 Nonlinear relaxation phenomena in metastable condensed matter systems Entropy 1920

[48] Spagnolo B, Valenti D, Guarcello C, Carollo A, Persano Adorno D, Spezia S, Pizzolato N and Di Paola B 2015 Noise-induced effects in nonlinear relaxation of condensed matter systems Chaos, Soliton Fract. 81412

[49] Pan F et al 2012 Proc. IEEE 70th Device Research Conf. (Universtiy Park, PA, 18-20 June, 2012) p 217

[50] Lanza M 2017 Conductive Atomic Force Microscopy: Applications in Nanomaterials (Weinheim: Wiley) p 384 
Experimental investigations of local stochastic resistive switching in yttria stabilized zirconia film

[51] Lanza M et al 2019 Recommended methods to study resistive switching devices Adv. Electron. Mater. 51800143

[52] Filatov D O et al 2014 Atomic Force Microscopy (AFM): Principles, Modes of Operation and Limitations ed H Yang (New York: Nova Science) p 335

[53] Seok J Y et al 2014 A review of three-dimensional resistive switching cross-bar array memories from the integration and materials property points of view Adv. Funct. Mater. 245316

[54] Lanza M 2014 A review on resistive switching in high-k dielectrics: a nanoscale point of view using conductive atomic force microscope Materials 72155

[55] Andronov A A, Belyakov A V, Guryev V A and Yakimov A V 2002 The interactive visual development of applications of automation scientific and industrial measuring and control systems by means of LabVIEW 6i National Instruments Proc. of the NATO Project SfP-973799 Semiconductors 2nd Workshop (Nizhnii Novgorod, Russia, 2002) p 38

[56] Horsthemke W and Lefever R 1984 Noise-Induced Transitions: Theory and Applications in Physics, Chemistry, and Biology (Berlin: Springer)

[57] Spagnolo B, Caldara P, La Cognata A, Augello G, Valenti D, Fiasconaro A, Dubkov A A and Falci G 2012 Relaxation phenomena in classical and quantum systems Acta Phys. Pol. 431169 